gum is not enough. Then, whether saliva or none at this early age be supplied, I know that by giving that child something to do, something for its jaws to act on, something that compelled it to labor for its hourly food, I but carried out that injunction that in "the sweat of thy brow thou shalt earn thy bread," and it was manifest on its brow in the perspiration from the labor in drawing the milk from the bottle. In this special case and in many others lives have been saved, for all hope had been abandoned until these nipples were used.

While on the case, I must speak of modified milk as its only food for nine months, which was changed from time to time to suit the babe, which acted all the time most charmingly, and with pure spring water as a drink from a similar nipple, nothing of artificial foods has entered its stomach since it came into my charge. It will be asked if it would not be well to chemically test the saliva? I say no! since my experience in operations in the mouth for forty-three years in adults would not let $m e$ do so to infants, as their saliva is about as pure as could be secreted. But of the adult's saliva $\bar{I}$ shall have much to say in a future article; how it is most powerfully affected both chemically and physically, and its diagnostic value as a factor in determining much that is wrong in the system. It is wonderful to note the many changes of saliva and how medicaments affect its odor and consistency. I pay much heed to the saliva in every case and the power it has over the hardest enamel of teeth, whether alkaline or acid, or laden with a profuse quantity of mucus, making it possible to spin it out like a thread of a foot in length.

As soon as time will permit I will report upon this subject of infant as well as adult salivary secretion, for no one could have been more impressed with its importance for health and, in connection with certain articles of diet and the proper use of the teeth in masticating food. I shall also speak of the injurious use of water and drinks of various kinds when food is being masticated in the mouth, and the necessity that the saliva should be kept carefully healthy when it is the only fluid used in masticating food.

The matter is of the greatest importance to health at all periods of life, and no secretion of the body can compare in value to the products of the parotid glands. With this skirmish over a most interesting subjeot I leave the matter, knowing that general investigation and observation will result in invaluable aid to infants who have, heretofore, had to go down to their death, and who now can without any doubt have the proper care and sustenance.

While the apparatus I have contrived is very simple, it is well worth the careful scrutiny of every lover of the innocent babe.

Gelatin Casts. - O. Vulpius of Heidelberg has a much admired collection of gelatin casts, which keep perfectly well in a dry place without shriveling, melting or growing musty. A plaster negative is taken of the part of surface desired, all grease removed, thoroughly dried and filled with the following mixture dissolved in the water-bath and partially cooled: colorless gelatin $250 \mathrm{gm}$., water $240 \mathrm{gm}$., glycerin $500 \mathrm{gm}$. To obtain a flesh tint rub up fresh together one part cinnabar, five parts zinc oxid and ten parts glycerin, and add six or eight grams to the gelatin. If the cast is to be very large it can be made with a thin layer of gelatin, and strengthened at the back with plaster when cold. The casts cool in twenty.four hours.-Cbl. $f$. Chir., February 5.

\section{GOUT OR RHEUMATISM,}

A REVIEW OF TREATMENT IN RELATION TO THE NORMAL, THE SENILE, THE ATROPHIO, AND THE NEURASTHENIC HEART.

BY J. J. CALDWELL, M.D., F.S.Sc., (London, Eng.) BALTIMORE, MD.

Gout ${ }^{1}$ is the name given to all those modifications of our metabolism caused by the gouty diathesis, as well as to all the symptoms to which those modifications give rise. Naturally, after middle life, gout affects every organ of the body both in its structure and its function. In a state of civilization we are all, after puberty, more or less gouty, and we are gouty in a gradually increasing ratio. All the various symptoms connected with the senile heart may be looked upon as indicating cardiac failure with sequential complications, and the treatment must therefore be tonic, with certain modifications. The drugs useful as cardiao tonics are but few in number, but of great value.

Digitalis is the foremost of all cardiac tonics; it gives its name to a whole group of remedies with similar actions, only one of which comes within a measurable distance of itself in the possession of valuable and reliable properties. An indigenous drug of the highest value, and known for more than a hundred years as a reliable remedy in dropsies, its action was so little understood, even so recently as twenty years ago, that it was called the opium of the heart, and was looked upon as a most powerful and dangerous sedative, and even yet the profession are more or less hampered in its use by an idea that digitalis is dangerously cumulative. Digitalis, like 'Fitz-James' blade," is both sword and shield, and he who understands its use will never be disappointed by it, the very so-called cumulative action being the necessary result of one of its most valuable properties when overdone.

Given in full doses at short intervals, digitalis, like many other drugs, is not wholly eliminated during the interval, but each succeeding dose reinforces those that have preceded till a dangerous degree of cardiac contraction does not seem to be dangerous if wittingly produced and carefully watched. It takes a good deal of digitalis to bring a human heart to a standstill in systole. Half-ounce doses of the tincture of digitalis used to be given safely and repeatedly in the treatment of delirium tremens. I have often given dram doses of the tincture every hour for four or five hours, in the precritical collapse of pneumonia, and many years ago, in treating the dilated heart of a young chlorotic girl, I kept her pulse for days at 40 , and her heart-sounds beating with the empty tic-tac of an infant's heart (embryocardia). In this case all my endeavors failed to contract and cure this dilated heart, which always relaxed the moment the dose of digitalis was reduced, apparently from sheer want of tone in the muscles. Persistent treatment failed to contract the heart, yet sufficed to feed it. It has kept well fed all these years, and, though a loud systolic murmur still indicates the continuance of dilatation, the patient has long been a happy wife and is the mother of several healthy children, with no appearance of any ailment about her.

What we were perforce reduced to in this case is all we should ever attempt in the case of senile hearts. We need never attempt to contract and cure a senile

1 Balfour's Senile Heart. 
dilated heart; it can not be done, so there is no use trying. But we always can improve the nutrition of the dilated myocardium, and in doing so we gain two ends-we fit the muscle for the more perfect discharge of its functions, and we enable it to better withstand injurious influences, reflex or other. With this object in view, we employ only moderate doses of digitalis, doses which never seem to have any cumulative action, or so rarely and slightly that we may safely continue them for a week or two without observation and with. out risk. These doses are from the British pharmacopeia preparations: The infusion-half a measured fluidounce; the tincture-ten minims. Each of these doses is equivalent to a little more than one grain of the powdered leaves, so that this may be taken as the medium dose that may be safely administered every twelve hours without risk of cumulative action. This means that within that space of time the quantity of the drug ingested has been completely balanced by that excreted, only the tonic influence remaining, that is, the improved nutrition of the myocardium, due to the action of the drug while being slowly excreted. have known such doses to be continued for many months, sometimes years. The dose of digitalis is not, however, an absolute one, but is relative to the bulk (weight) of the individual, and especially to the amount of his blood, a weakly anemic individual tolerating a very much smaller dose than one more plethoric. Now and then we come across an idiosyncrasy which either tolerates freely a larger dose or resents any but the smallest; such cases are, however, rare. Still, in view of their occasional occurrence, it is well that a patient under treatment for the first time should be seen now and then for the first week or two; afterward when the measure of toleration, as we may term it, has been ascertained, this may be less necessary.

The object we have in view when using digitalis in case of senile heart is not to remove dropsy, to slow the rate of pulsation, or to contract the cardiac cavities, but by the gradual accumulation of trifling advantages, to tone up and strengthen the cardiac muscle by improving its nutrition. Gradually the heart acts with more vigor, the circulation improves in steadiness and force, the edema occupying the tissue spaces is removed, and thus the blood-pressure is lowered and a considerable strain taken from the heart. For this purpose only moderate doses are required, doses which can be continued for many months with. out any risk of dangerous accumulation, and yet which have a decided effect in strengthening the heart, im. proving the tone and elasticity of its muscle, and accumulating energy in its ganglia. Naturally this process is a slow one, and the benefit is not, for a time, very obvious to the recipient. Some years ago a friend called on me and said: "Doctor, your medicine is doing me no good." "Of that," I said, "you must allow me to be the best judge." "But I feel no change in my symptoms, nor any action whatever from what you have given me." "I had expected nothing else; you are too impatient," I replied, "and were I to give you medicine in such a dose as to produce a sensible action in a few days, before long its action would be so unpleasant that you would either stop it yourself, or your ordinary medical attendant would order you to give it up. In a short time the seeming benefit would vanish, and you would abuse me for having given you medicine which did not agree with you, and which gave you no permanent relief. Now, what I have given you will not speedily relieve you, but give it time, and it will make you well and prolong your days in comfort. Two or three months after this you will say to your wife some morning, 'Do you know my heart is not so troublesome as it was; I begin to think I am improving;' and six or eight months after this you will come to me and say, 'Doctor, I was preaching last Sunday, and feel none the worse for it." And so it fell; my friend and his senile heart are nowadays, after the lapse of five years, still very good company to each other, which for many days they were not.

The senile heart owes its peculiar symptoms and progress to the difficulty which a weakened myocardium finds in maintaining the circulation in the face of the permanent obstacle presented by rigidity of the arterial walls. To seek to excite a heart to more powerful action, in the face of such an obstacle, seems fraught with danger, and we know that even moderate digitalis stimulation in such circumstances is apt to be followed by an increase of the symptoms, sometimes by an increase of the cardiac dilatation, and always of its erethism. Some also object to the use of digitalis when the arteries are atheromatous, from a dread of rupturing their brittle coats. This lastnamed danger must be a very infinitesimal one, as such an accident is quite unknown to me, notwithstanding a continual and free use of digitalis. But, indeed, the same means of necessity taken to prevent the increase of cardiac erethism would also prevent this more serious danger. To this end it is needful in all such cases to combine the digitalis with some drug capable of unlocking the arterioles, such as iodid of potassium, the nitrates of which-nitrous ether, nitrite of sodium and nitroglycerin-are those most commonly used.

Digitalis should never be prescribed in a case of senile heart without the addition of one or more of these vascular stimulants; iodid of potassium is the most useful, acting well and persistently in a moderate dose, and being free from any objectionable effect. If, at the commencement of the treatment, the heart has been much neglected, the dilatation considerable, and the irregularity great, it is very desirable to begin with larger doses of digitalis than those just recommended so as to gain control over the rate and rhythm of the heart as rapidly as possible; but these large doses are not likely to be required for any length of time, and ought to be intermitted for at least a couple of days before the patient is put upon the smaller doses for a continuance. Where there is edema of the lower limbs, a perfectly dry diet with tonic doses of digitalis are quite often sufficient to remove the fluid, and that in a very short time; but if the dropsy is at all considerable, it must be treated as an ordinary case of cardiac dropsy, and in such cases it is a great saving of time to drain the limbs. In all senile hearts, whatever their character or special symptoms may be, we must always remember that digitalis uncombined with one or the other of the vascular stimulants is never so beneficial as when it is so combined; otherwise it is certain to produce discomfort and likely to do serious damage. The only other member of the digitalis group which has any pretensions to rival digitalis itself is strophanthus and its active principle - strophanthin. Strophanthus is, however, so much more uncertain in its action, especially as to its feeding or tonic properties, than the leading member of its group, that I have never felt 
inclined to displace our own pre-eminent and indigenous drug in its favor.

Strophanthin, however, possesses two advantages over digitalis-it is readily soluble in water, and it seems to act with great rapidity. There are, therefore, conditions in which strophanthin is to be preferred, but these are unusual and exceptional at all times, and are rarely found in connection with the senile heart. Nux vomica is an excellent tonic for the senile heart and its concomitants, but, as its usefulness depends upon its active principle, it is more advantageous, and contributes to greater accuracy of dosage to employ liquor strychnin-hydrochlorate, or preferably nitrate - rather than any of the other crude preparations. The maximum benefit is only to be had from any other drug by using the maximum dose for a sufficient length of time, and to do this safely with any remedy, but, especially with so powerful a drug as strychnin, it is needful to be both accurate in the doses and regular in the times of administration. Strychnin is cumulative in its action, but by strict adherence to the rules laid down, it may be used continuously and safely for many years. I have known five minims of liquor strychnin-1/26 grain of strychnin-to be taken twice a day for over ten years, with the best results. At the end of that time symptoms of saturation began to appear, and the strychnin had to be discontinued, as it was no longer required, the puny, delinate, middle-aged woman being now both strong and bealthy. It is only rarely that a larger dose than five minims of liquor strychnin can be given daily with benefit. Three such doses, fifteen minims, instead of ten, in the day are generally followed by symptoms of poisoning in no long time.

Idiosyncrasy occasionally turns $u p$, and for the larger number of mankind may be for any length of time. In anemic patients there is often intolerance of strychnin, and if employed at all it has to be given in almost infinitesimal doses. Strychnin acts in two ways -it is an admirable tonic for the stomach, especially in those catarrhal conditions accompanied with venous congestion so commonly present when the circulation is feeble. In this way the digestion is improved and the blood enriched, so that the body generally, and the heart in particular, gets better nourishment. Strychnin has also a specially stimu. lating effect on the nervous system generally; consequently it is stimulating and renders more excitable the vasomotor center and the cardiac ganglia, probably energizing the primordial powers of spontaneous movement possessed by the cardiac muscular fiber, vis in situ, the once diffuse nervous force. In virtue of this action on the heart and nerve-centers, strychnin increases the cardiac force, revises the intra-arterial blood-pressure, and is, next to digitalis, the most elegant tonic we possess for all feeble and dilated hearts. In the less serious class of cases it is sufficient of itself to give tone boin to the heart and the system generally, while in the most serious cases it is the most useful adjunct to digitalis.

Arsenic or arsenious acid (1/50 grain) is another of our most valuable tonics. It is advantageously employed in many forms of disease, and it is quite indispensable in the treatment of the senile heart. It is useful in the congestive conditions of the stomach which accompany cardiac failure, and its effect in angina is sometimes almost magical, the suffering disappearing like a dream, quite apart from any influence exerted on the cardiac failure, upon which that suffer- ing seemed to depend. Masselot and Trousseau have both remarked upon the increased capacity for exercise that follows the administration of arsenic, and this doubtless depends upon the same general tonic influence affecting the lungs, heart and blood, that makes breathlessness a thing unknown to the Styrian mountaineer, and restores the bloom in the aged, and freshness of youth to old and seemingly worn-out horses. The Styrians are accustomed to take large doses--as much sometimes as five grains-of pure arsenious acid in the day, but such dangerous doses are by no means necessary to obtain the tonic benefits of the drug. Most excellent resulte occasionally follow the prolonged uses of almost infinitesimal doses (1/50 grain). I well remember one old gentleman, exceedingly sensitive to the action of drugs, to whom $1 / 50$ grain of arsenious acid was quite poisonous, but who could tolerate $1 / 100$ grain without difficulty. After taking this minute dose daily for two or three weeks, and nothing else, for a dilated and hypertrophied heart beginning to fail, he said to me: "I don't know what benefit you expect from the treatment, but I know what I have received; I can go upstairs much easier than I used to do." Arsenic may be given alone, and in anemic and very sensitive patients, who can only tolerate a very minute dose, this is often the best way of employing it. To these, one granule of arsenious acid containing $1 / 50$ or $1 / 100$ grain (or a tablet triturate) may be given after food once or twice a day and for many months, with increasing benefit. More usually, it is better to combine the arsenio with digitalis or strychnin, or with both. We learn from the histories of the Styrian arsenic-eaters, that arsenic is a poison to which the system may be gradually habituated so that even large doses may be taken for many years, not only with impunity, but with positive benefit. When given, therefore, in the moderate medical dose of two or three minims of Fowler's solution, we may safely continue them twice a day for as long as we think needful without any misgiving periods, nor need we have any dread of danger in leaving off the drug after a long continuance, as was at one time alleged. A little caution may be required in commending its use, as idiosyncracy plays a marked part in relation to arsenic, but it is only rarely that we fall in with those who are extra-sensitive to its action.

When the blood is deficient in hemoglobin, iron is a necessity. It is best given along with food, and should never be combined with digitalis, as such a combination is very apt to sicken. The proto-salts ${ }^{2}$ of iron are to be preferred to the per-salts, as they are more easily taken up by the acids of the gastric juice, and are thus readily assimilated. As a rule, large doses are not required in cases of feeble or dilated heart. Intra-arterial blood-pressure depends upon the distension of the arterial system by the blood contained within it. This vasuular turgescence in its turn depends upon the relation between the amount of blood pumped into the arteries by the heart and the outflow through arterioles. After middle life the out. flow through the arterioles is hindered by obsolescence of the capillaries and by loss of arterial elasticity, and the blood-pressure is raised by the obstacles, even with the heart beating at its normal rate and force. A healthy heart has sufficient reserve force to enable it to cope successfully with the demands for extra exertion thus made upon its powers, and it thrives upon its exertions; but when the heart is from any 
cause feeble and ill-fed, it fails to respond, and it suffers. Its sufferings give rise to those varied symptoms comprised under the term "senile heart."

As these sufferings are caused and maintained by the high blood-pressure, whatever lowers this always gives relief; hence these sufferings are capable of being relieved by various modes of treatment, not all of them being truly remedial. To permanently remove these sufferings, w $\theta$ must not content ourselves with merely reducing the blood-pressure, but we must also strengthen the heart so as to enable it to cope with a blood-pressure always above the normal of adolescence, and which is liable to be suddenly abnormally raised by many causes. Cardiac tonics are therefore required, but all cardiac tonics, except, perhaps, arsenic, are also cardiac stimulants, as they increase the elasticity and contractility of the heart, and in certain conditions they improve the heart's metabolism by enabling it to feed. itself with a larger blood-wave at a higher pressure. When the heart is feeble, however, this is just what can not be done. The whole trouble has arisen because the blood-pressure is already too great for the powers of the heart, and if we goad this feeble organ to further exertions, for which it is unfit, we either increase the dilatation that may be present, or induce erethistic tachycardia or irregularity. Cardiac tonics do not agree, but we can make them agree by combining a vascular stimulant with a cardiac stimulant, thereby making things work smoothly. The heart, no longer opposed by an obstacle it can not overcome, or only imperfectly, feeds itself better, and all its sufferings vanish.

Vascular stimulants are agents which dilate the peripheral vessels (arterioles), and so promote the flow of blood from the arteries into the veins and lower the intra-arterial blood-pressure.

Iodid of potassium is not perhaps generally regarded as a vascular stimulant, but in so far as it promotes the flow through the arterioles and lowers the blood-pressure it is an eminent member of that group, as has been established experimentally and duly recognized in relation to the treatment of aneurysm. It is not rapid in its action, but it is persistent, two or three grains every twelve hours being quite sufficient to enable digitalis to be given freely without any cardiac disturbance.

All the nitrites are vascular stimulants; spirits of nitrous ether or nitrite of sodium may be so employed, but their action does not last so long as that of the iodid of potassium, while in rapidity they are far inferior to either the nitrite of amyl or nitroglycerin. Besides being a vascular stimulant with all the actions belonging to such remedies, nitrite of amyl is also an analgesic. A former patient, who suffered from intense pain accompanied by a large aortic aneurysm, from which he died, always found the analgesic action absent unless the drug was freshly prepared. When not quite fresh, his face flushed, and all the usual symptoms due to amyl were produced, but the pain was not relieved. Since then the amyl has been retailed in hermetically sealed glass capsules, apparently with the effect of retaining the analgesic properties. The flush of the face and the fulness of the head and rapid action of the heart produced by the amyl are disagreeable to some patients, but as they do not seem to be in any way injurious, I have known amyl to be used with great freedom in angina.

2 Solution of Iron-Manganese (Gude). medical friend, who suffered much from angina, connected with aortic regurgitation, not content with inhaling it frequently during the day, used to soak his pocket handkerchief in the amyl, and go to sleep with it lying on his face, without any ill results. The action of the amyl is very evanescent, the smell is disagreeable, and the quantity in a single capsule is rather small, but that is a matter easily remedied. Nitroglycerin, or trinitrin, is said to be a nitrite of glyceril, but its action is that of a nitrite in ordinary medical doses of $1 / 50$ to $1 / 100$ grain.

The importance and advantage of paying attention to the diet and feeding of bronchitic and asthmatic patients ${ }^{3}$ has been impressed upon me from more than one point of view, and in these few remarks I wish to offer some suggestions that may prove useful. I have often noticed that when a patient, who has been long ill with chronic bronchitis and emphysema of the lungs, dies suddenly, it has almost always been soon after partaking of a full meal. I have met with instances among hospital out-patients where a man, after a heavy day's work in cold, foggy weather, has come home to a hearty tea or supper, and afterward has been taken with breathlessness, quickly followed by cardiac failure and death by his own fireside. On the other hand, I have come across elderly patients, greatly afflicted with asthma and chronic bronchitis, who, either in obedience to the doctor's orders, or from some instinct of their own, have modified and cut down their diet, with such relief to their breathing powers as has been most remarkable.

That stimulants are invaluable in cases of acute heart failure fow will deny, but the regular administration of alcohol night after night, often in potency, is pretty sure to cause congestion of the liver and overfulness of the right auricle, conditions powerfully conducive to heart palpitation and breathlessness. Many farinaceous foods, wholesome enough in themselves, are often taken in such large quantity and in such injudicious combination that they set up flatulent fermentation in the stomach and cause distressing acidity and heartburn. This is especially the case with elderly people who have slow digestion. The distended stomach rises up against the heart, impeding, and sometimes permanently stopping, the action of this organ. The patient will declare that all he takes is most wholesome. Breakfast perhaps consists of a great soup-plate full of porridge, followed by eggs and mutton chops. The last meal of the day is made up of some bulky farinaceous food with roasted apples and sometimes lemonade, sometimes a glass or two of claret as a drink.

In the treatment of such a case the doctor has his opportunity. He will look at his patient's tongue and will find it large, swollen, and indented by the teeth; it is more or less furred and the breath is sour. The urine is often copious, clear, and frequently passed. There probably is never any gastric pain or tenderness, the stomach being in a dull and torpid condition, yet constantly craving for food. Good may here be done by banishing the plate of porridge from the breakfast table, and allowing one egg with a thin slice or two of bacon, and rusks or dry toast in place of bread. As a change, fish, fresh or salted, suits well, and salt herrings are reckoned good for the throat. At the midday meal roast meat is proper, with such vegetables as are found to agree. Light puddings, in

3 Thorowgood, London Hospital for Diseases of the Chest; Medical Press and Circular, Dec. 16, 1896. 
moderate quantity, are allowable, and often a glass or two of sherry appears to aid the digestion.

The most important part of this dietetic treatment consists in the arrangement of the evening meal. This should be taken not later than 8 P.M., and must be light, digestible and nutritious. Soups as a rule do not agree, especially when the heart is feeble. Chicken or game seems to suit most people. Ham and tongue in thin slices also agree well. Beef, and even mutton, late in the day, are often too much for some stomachs.

Dr. W. H. Thomson believes that the treatment must be determined chiefly by the consideration whether the affection be primary or secondary in its causation. In the treatment of primary affections the first indication is the prophylaxis of rheumatism. Particular attention should be paid to the tonsils, and prompt use of the salicylates internally should be made at the first signs of tonsillitis, together with local applications of tincture of iodin. The habitual employment of hot-water douches, with oil of peppermint, to the throat is an excellent measure. Rheumatic patients should always protect the skin with flannel, both by day and by night. Rest in bed is of great importance in the primary affections. When the symptoms are rather aggravated by digitalis, strychnin, and similar drugs, aconite will often leszen the dyspnea, relieve the anginose attacks, and make the pulse fuller and steadier. In dyspnea, the result of pericarditis, firm strapping of the right side of the chest is of undoubted service. For the dyspnea of mitral stenosis, especially when it occurs in paroxysms, belladonna in combination with compound spirits of ether is much the best remedy. In mitral stenotic cases, digitalis is often inferior to strophanthus, spartein, or caffein. Anemia should always be considered, but the administration of iron is mischievous in rheumatism. In this disease the best remedies are cod-liver oil with small doses of arsenic; or calcium sulphid in from one-half to one-grain doses four times daily apparently hastens the convalescence from acute rheumatism. In secondary disease of the heart one should consider the whole extent of the circulation. The remedial agents may be divided into the constitutional and symptomatic. Of the first class, and the most important and continuous in its operation, is fresh air. Iron is the most effective among drugs, because it is our respiratory food, and has no other funotion in the body but as the carrier of oxygen. Next to continuous breathing in the open air is properly applied massage, especially that form in which the patient exerta a carefully regulated resistance to the flexion and extension of his muscles by the operator.

Acute alcoholic dilatation of the heart is a most definite, important, and striking affection. Two very instructive non-fatal cases were described by Dr. Maguire in "The Transactions of the Clinical Society," London, Vol. xxii, 1887.

The following fatal case, ${ }^{4}$ which came under my notice a few weeks ago, illustrates the disease in a very striking manner:

A man, aged 30 years, by occupation a fitter, was admitted into the General Hospital, Nottingham, Oct. 26, 1896. He had slight general edema, great swelling of the legs, a distended abdomen, and such a pale, pasty face as to strongly suggest chronic nephritie, which had been the provisional diagnosis made in the out-patient room by the house physician before any urine could be obtained for examination.

4 Medical Press and Circular, London, England, March 24, 1897.
On admission he was drowsy, and evidently very ill; but his statement was clear that he had never had rheumatic fever, or any previous serious illness, and had been quite well until b had got out of work eight weeks before. The urine was tested when I saw him-an hour or two after admission-and was found to be scanty, sp. gr. 1022, and perfectly free from albumin. He complained of failing sight; and on ophthalmoscopic ex amination the discs were a little hazy, and the fundus very pale-possibly there was very slight edema of the retina. It was afterward ascertained from his mother that he had been drinking spirits very excessively for a month, and taking scarcely any food. The pulse was rapid, 100 to 120 per minute, of very low tension, and exceedingly feeble. The area of cardiac dul ness was increased, but no cardiac impulse could be defined. There was no murmur, and the sounds were very weak. Although in some forms of chronic Bright's disease the urine may be found free from albumin, sometimes for long periods, the above complex of physical signs was incompatible with nephritis, acute or chronic. The diagnosis was cardiac dilatation and feebleness. The patient somewhat suddenly became cya osed at 6 A.M. the following morning, and died at 10 A.M.

Necropsy, seven hours postmortem: Tissues edematous; a little fluid in the peritoneum and pleura; three ounces in the pericardium. The right auricle was considerably dilated and the walls thickened; the right ventricle was much dilated and the walls considerably thickened; they were soft in texture, and there appeared to de some fatty degeneration of the mus cular substance. The left auricle was rather less dilated than the right, and the left ventricle more dilated than the right.

All the cavities were distended with soft, recent, black clot; and all the valves were healthy. The coronary arteries appeared normal ; the aorta was small, and just spotted with commencing atheroma. The weight of the heart, opened and well washed, was $14 \frac{3}{4}$ ounces; the man was very slightly built, height about 5 feet 2 inches. The kidneys were quite healthy to the naked eye; the capeules stripped easily, the cortex was in normal proportion; they weighed together seven ounces. The liver was rather pale and fatty.

The only marked pathologic condition, therefore, was a decided dilatation and enlargement of the heart.

The above may be recapitulated ad libitum, for their names are legion in the practice of every busy physician.

In conclusion, I will say that I have many cases of heart trouble illustrating the benefit and variety of the above-named treatments, which will appear in my second paper of clinical reports on "The Treatment and Therapeutics of Gout or Rheumatism and Inebriety in Relation to the Heart."

\section{METALLIC MERCURY.}

ITS USE IN THE FORM OF UNGUENTUM HYDRARGYRI OR BLUE OINTMENT ADMINISTERED BY THE MOUTH.

Read before the Mississippi Valley Medical Association, at Nashville, Tenn., Oct. 11-14, 1898.

BY ALBERT BERNHEIM, A.M., M.D.

PHILADELPHIA, PA.

The purpose of this paper is not to speak on the value of blue ointment for itself, in the treatment of syphilis; there is no doubt about its great and quick efficacy, quite especially where an immediate result is desired. In general, blue ointment was always used by the method of inunction, but very often this method of administration is disliked by the patient, on account of its nastiness and inconvenience. If we can substitute another method of using it, with the same good results, I think it would be of no small advantage.

It was Dr. Anuschat, a German physician, who, in 1896, recommended the internal administration of blue ointment. He started from the view that, as is well recognized at the present time, the efficacy of the inunction of blue ointment depends upon the evaporation of the metallic mercury. I am very well aware that not all the investigators agree in regard to the manner of action of blue ointment. On the one side, the authors say that the globules of the metallic mer- 